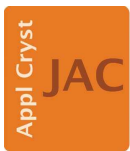

JOURNAL OF

APPLIED

CRYSTALLOGRAPHY

ISSN 1600-5767

Received 28 November 2014

Accepted 5 April 2015

Edited by D. I. Svergun, European Molecular Biology Laboratory, Hamburg, Germany

Keywords: small-angle X-ray scattering; nanocrystals; shape retrieval; DAMMIN; DAMAVER.

Supporting information: this article has supporting information at journals.iucr.org/j

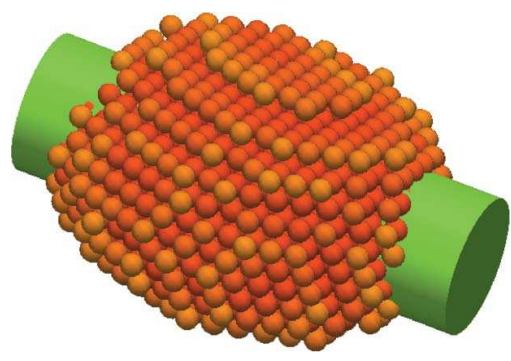

C 2015 International Union of Crystallography

\section{Considerations on the model-free shape retrieval of inorganic nanocrystals from small-angle scattering data}

\author{
Max Burian, ${ }^{\mathrm{a}, \mathrm{b} *}$ Gerhard Fritz-Popovski, ${ }^{a}$ Meng He, ${ }^{\mathrm{c}, \mathrm{d}}$ Maksym V. Kovalenko, ${ }^{\mathrm{c}, \mathrm{d}}$ \\ Oskar Paris $^{\mathrm{a}}$ and Rainer T. Lechner ${ }^{\mathrm{a}}$
}

a Institute of Physics, Montanuniversitaet Leoben, Franz-Josef-Strasse 18, A-8700 Leoben, Austria, ${ }^{\mathbf{b}}$ Present address: Institute of Inorganic Chemistry, Graz University of Technology, Stremayrgasse 9, A-8010 Graz, Austria, 'Department of Chemistry and Applied Biosciences, ETH Zurich, Vladimir Prelog Weg 1, CH-8093 Zurich, Switzerland, and ${ }^{\mathbf{d} E m p a-}$ Swiss Federal Laboratories for Materials Science and Technology, Ueberlandstrasse 129, CH-8600, Duebendorf, Switzerland. *Correspondence e-mail: burian@tugraz.at

Some new considerations on the model-free shape retrieval of inorganic nanocrystals based on the evaluation of averaged dummy atom models obtained from small-angle X-ray scattering data are presented. Scattering curves for shapes commonly found in inorganic nanocrystal systems were computed, from which dummy atom models were reconstructed using DAMMIN and $D A M A V E R$. Cross sectional analysis methods were applied to allow a quantitative interpretation of these models, where special focus was directed towards the measurement of discrete model dimensions. By a quantitative comparison of the obtained models with the initial geometries, the limits of the proposed evaluation techniques were tested. Further, the proposed methods were utilized to study the influence of the accessible scattering vector as well as the effect of increasing size distributions on models retrieved by DAMMIN and $D A M A V E R$. The results confirm the usefulness of these ab initio shape-retrieval methods for slightly polydisperse systems. Finally, the practicability of the proposed techniques is demonstrated on an ensemble of chemically synthesized colloidal bismuth nanocrystals.

\section{Introduction}

In recent years, inorganic colloidal nanocrystals (NCs) have increasingly received attention because they are promising for various applications such as for electronic devices and solar cells (Robel et al., 2006; Gur et al., 2005; Nair et al., 2011) or light-emitting diodes (Halpert et al., 2008; Reiss et al., 2009; Rogach et al., 2008). One of the significant attributes of NCs is that their unique physical and chemical properties strongly depend on their size and shape (Hens et al., 2002; Wehrenberg et al., 2002). Advances regarding the chemical synthesis of metal and semiconductor NCs allow the explicit tuning of these parameters up to a degree that complex polyhedral shapes (Huang \& Lin, 2012) as well as core-shell structures (Reiss et al., 2009; Lechner et al., 2014) can easily be achieved. But, with this increasing level of structural complexity, greater attention has to be directed towards the development of methods capable of resolving such morphologies. Transmission electron microscopy (TEM) is the most common method used to achieve this because images with single-atom resolution can easily be obtained. However, this technique has some major drawbacks. In particular, TEM images can only be obtained within the focusing plane of the microscope. This implies that the $\mathrm{NC}$ shape along the direction perpendicular to the focusing plane cannot be observed. While this issue can 
partly be avoided using TEM tomography (Ahrenkiel et al., 2008; Midgley \& Weyland, 2003), high-quality TEM images that contain information about the NC shape can only be made of a few hundred NCs at once, whereas most NC devices consist of $10^{6}$ or more NCs.

To retrieve average structural parameters from a large ensemble of nanoparticles, the method of choice is small-angle scattering (SAS). Over the past decade, various shaperetrieval methods using SAS data have been established as powerful tools in a range of scientific communities. The ability of SAS experiments to obtain three-dimensional shape information from a very high number of particles which are oriented in different directions is a big advantage, this being the overall challenge of all shape-retrieval methods. Particles in solution imply, in particular, that only the so-called orientational averaged SAS signal can be measured. A common approach to tackle this issue is to assume some kind of particle shape, which allows the fitting of a given (analytical or numerical) form factor (e.g. Alves et al., 2014) to an experimentally obtained scattering curve by varying the initial model parameters. This procedure implies, however, some kind of $a$ priori knowledge of the system, such as the particle shape from, for example, TEM tomography. One of the landmarks in the development of shape-retrieval methods was the introduction of ab initio methods such as DALAI_GA (Chacon et al., 1998), DAMMIN (Svergun, 1999) and SAXS3D (Walther et al., 2000). These programs are all based on the well known Debye formula (Debye, 1915), which allows the calculation of the scattering intensity of a given assembly of spheres [also called dummy atoms (DAs)]. By iteratively changing the initial random assembly and comparing its scattering intensity with the experimental one, a threedimensional so-called dummy atom model (DAM) describing the particle shape can be obtained. If this fitting procedure is repeated numerous times, the resulting DAMs can be used to calculate an averaged dummy atom model (aDAM) (program DAMAVER; Volkov \& Svergun, 2003), which initially was suggested as a tool to investigate the stability of the fitting solution. These programs are presently predominantly applied for the determination of protein low-resolution quaternary structures. Both the single simulation and the aDAMs represent a substantial source of information from which orientational and positional relationships between the macromolecules can be deduced. Furthermore, if macromolecular structures of domains or subunits are known, the obtained DAM can be used for rigid-body modelling or refinement (Koch et al., 2003; Trewhella et al., 2013). A good overview regarding $a b$ initio methods and their practical use is given by Blanchet \& Svergun (2013). The largest disadvantage of $a b$ initio methods is their limitation to strictly monodisperse systems such as proteins. To our knowledge, only two studies have been published on the potential use of the $a b$ initio shape-retrieval method DAMMIN on slightly polydisperse systems (Shtykova et al., 2007, 2012). In contrast to proteins, crystalline nanoparticles feature two major differences: (i) they always exhibit a certain amount of polydispersity and (ii) they may contain facets and edges due to anisotropic surface energies and due to elastic misfit interactions. However, the majority of inorganic nanocrystals show highly symmetric shapes such as spheres, cubes or polyhedra because crystals will, in general, minimize their Gibbs free energy and, therefore, minimize their total free surface energy. If the particles exhibit isotropic or only slightly anisotropic surface energies, the overall equilibrium shape will, therefore, be close to spherical.

In this work. we study the use of DAMMIN and $D A M A V E R$ for model systems similar to those found in inorganic NCs. Scattering curves for simple mono- and polydisperse geometries such as spheres, cubes, ellipsoids of revolution and spherical core-shell structures are computed, from which DAMs are obtained. To provide measures for particle dimensions from the resulting aDAMs, we present data evaluation tools based on spherical cross sectional analysis procedures that allow a qualitative and quantitative description of the models. The evaluation methods shown are validated and utilized to discuss the effects of varying simulation parameters. In particular, the influence of smearing effects due to size distributions on the obtained DAMs is investigated. As an application example, the presented methods are applied to retrieve the mean shape of a large ensemble of diluted Bi NCs with high accuracy.

\section{Methods}

The scattering intensity measured in a typical small-angle $\mathrm{X}$-ray scattering (SAXS) experiment of monodisperse homogeneous particles, such as colloidal NCs that show no interaction, can be written as

$$
I_{\text {exp, mon }}(q)=N \Delta \rho^{2} V^{2} P(q),
$$

where $q$ is the scattering vector modulus [defined as $q=(4 \pi / \lambda) \sin \theta$ with the scattering angle $2 \theta$ and the wavelength $\lambda], N$ is the number of probed particles, $\Delta \rho^{2}$ is the electron-density difference between the solvent and the particle, and $V$ is the particle volume. $P(q)$ [defined as $P(0)=$ $1]$ is the spherically averaged form factor of a given particle, which describes the scattering that can be attributed to the particle shape, homogeneity and size. Equation (1) is based on the assumption that the NCs are oriented in all possible directions such that only the spherical averaged signal is calculated. For the shape retrieval of perfectly monodisperse systems the scattering intensity can simply be calculated from the form factor as $N ; \Delta \rho^{2}$ and $V^{2}$ merely cause a constant scaling of the scattering intensity.

For the polydisperse systems presented in this work, an assumption was made that polydispersity only affects the particle size and not the shape. The size distributions were described using Gaussian volume distributions from which the corresponding number distributions $N\left(r_{i}\right)$ can be calculated. For the example of homogenous spheres, equation (1) can be rewritten such that the scattering intensity reads 


$$
I_{\text {exp,pol }}(q)=\Delta \rho^{2} \sum_{i=0}^{M} N\left(r_{i}\right) V\left(r_{i}\right)^{2} P_{\mathrm{sph}}\left(q, r_{i}\right),
$$

where $M$ is the number of points $i$ used to describe the number distribution and $r_{i}$ is the radius of the sphere at a given point. The spherical form factor $P_{\mathrm{sph}}(r, q)$ can further be calculated using the square of the scattering amplitudes $F_{\text {sph }}(r, q)$ according to

$$
F_{\mathrm{sph}}(r, q)=\frac{3[\sin (q R)-q R \cos (q R)]}{(q R)^{3}} .
$$

Additionally, in this work we present model scattering data from systems of polydisperse ellipsoids, in which case the form factor also has to be calculated numerically. For an oblate ellipsoid, the semi-axes $a, b$ and $c$ can be described such that $a=b=R$ and $c=\varepsilon R$, where $R$ is used as a reference dimension. The form factor can then be calculated by a numerical average over the angular differential $\mathrm{d} \alpha$ according to

$$
P_{\mathrm{ell}}(q, R, \varepsilon)=\int_{0}^{\pi / 2} F_{\mathrm{sph}}^{2}[q, r(R, \varepsilon, \alpha)] \sin \alpha \mathrm{d} \alpha,
$$

where $r(R, \varepsilon, \alpha)=R\left(\sin ^{2} \alpha+\varepsilon^{2} \cos ^{2} \alpha\right)^{1 / 2}$ (Pedersen, 1997). The model scattering intensity can be calculated in analogy to equation (2) by replacing $P_{\mathrm{sph}}\left(q, r_{i}\right)$ by $P_{\text {ell }}\left(q, r_{i}, \varepsilon\right)$ while keeping the semi-axes aspect ratio $\varepsilon$ fixed.

The initial form factors and, therefore, the initial scattering intensity $I(q)_{\text {init }}$ of all cases presented in this work were computed using the programs GIFT (Bergmann et al., 2000) and SASfit (Kohlbrecher, 2008). A statistical error band with a mean deviation of $\sigma=\left[I(q)_{\text {init }}\right]^{1 / 2}$ was calculated for each data point. All data points were randomly distributed within the error band to distort the ideal scattering intensity. The range of scattering angles was described using Shannon channels (SCs) of width $w=\pi / D_{\max }$, where $D_{\max }$ is the largest particle dimension (Shannon, 1948). Unless stated otherwise, $q_{\min }$ was chosen for all initial scattering curves to be within the first SC, whereas $q_{\max }$ is within $15-20 \mathrm{SCs}$, as suggested in earlier work (Moore, 1980; Volkov \& Svergun, 2003). Pair distance distribution functions $p(r)$ (Glatter, 1977) were calculated from the initial scattering curves using the indirect transform method GNOM (Svergun, 1992). The scattering intensity from the model particle shape is then fitted by the ab initio shaperetrieval software DAMMIN (Svergun, 1999), using a simulated annealing procedure (Kirkpatrick et al., 1983). In the first step, an initial search volume is randomly filled with identical beads, from which the scattering intensity $I(q)_{\text {DAM }}$ can be calculated using the Debye formula (Debye, 1915). By iteratively changing the bead configuration and comparing $I(q)_{\mathrm{DAM}}$ with $I(q)_{\text {init }}$ a most probable configuration is found where the shape of the object is represented by the final DAM.

The maximum dimension $D_{\max }$ obtained from the $p(r)$ of the initial scattering curve is used to calculate the initial search volume such that its diameter is $1.1 D_{\max }$ (Volkov \& Svergun, 2003). DAMMIN was run in interactive mode, where neither symmetry restrictions were applied nor values other than the default were used for looseness, disconnectivity and periph- eral penalty weight (Svergun, 1999). Unless stated otherwise the maximum order of spherical harmonics $(L=20)$ was used. The packing radius of the DAs was chosen to result in approximately 6000 DAs within the initial search volume. To ensure acceptable fitting results in the higher $q$ regime, logarithmic curve weighting was selected. All DAMMIN simulations were repeated ten times to compute an aDAM using $D A M A V E R$, which is explained in more detail by Volkov \& Svergun (2003). During the averaging process, all DAMs obtained from DAMMIN are aligned, filtered for significant outlying DAMs and superimposed on top of each other using SUPCOMB (Kozin \& Svergun, 2001). Subsequently, a new densely packed DA grid is imposed. For every new grid position $\mathbf{x}_{i}$ the so-called occupancy $\operatorname{occ}\left(\mathbf{x}_{i}\right)$ can be calculated, which is the number of DAs of all superimposed DAMs in the vicinity of the new grid point. To our knowledge, the interpretation of such aDAMs is usually made rather qualitatively, as they are only used as input for manual rigid-body modelling (Svergun et al., 2001; Marquez et al., 2003). For instance, if DAs with occupancy below a certain threshold value are neglected, the most populated volume (MPV) is formed (result of the DAMFILT subroutine of DAMAVER). Even though the MPV preserves the main characteristics of the particle shape, no attention has yet been directed towards the detailed occupancy distribution because the aDAM is only described using the bead positions of the remaining DAs and not using the actual occupancy values.

In this work, the occupancy of all given points was normalized to the maximum occupancy value found in the aDAM such that $\operatorname{occ}^{*}\left(\mathbf{x}_{i}\right)=\operatorname{occ}\left(\mathbf{x}_{i}\right) / \operatorname{occ}_{\max }$. In analogy to standard single-body mechanics, the centre of mass $\mathbf{X}_{\text {com }}$ of the aDAM was calculated using the normalized occupancy as a virtual weight of the DAs such that

$$
\mathbf{X}_{\mathrm{com}}=\sum_{i}^{N} \mathbf{x}_{i} \operatorname{occ}^{*}\left(\mathbf{x}_{i}\right) / \sum_{i}^{N} \operatorname{occ}^{*}\left(\mathbf{x}_{i}\right)
$$

The aDAMs were then repositioned within the grid to place $\mathbf{X}_{\text {com }}$ at (000).

\subsection{Weighted radius of gyration}

For a known particle shape with a continuous electron density distribution $\rho(\mathbf{r})$ and the centre of mass at the origin of the Cartesian system, the radius of gyration $R_{\mathrm{g}}$ can be calculated as (Rubinstein \& Colby, 2003)

$$
R_{\mathrm{g}}^{2}=\frac{\int \rho(\mathbf{r})|\mathbf{r}|^{2} \mathrm{~d} V}{\int \rho(\mathbf{r}) \mathrm{d} V} .
$$

For discontinuous electron density distributions, equation (6) can be rewritten as

$$
R_{\mathrm{g}}^{2}=\sum_{i}^{N} q_{i}\left|\mathbf{r}_{i}\right|^{2} / \sum_{i}^{N} q_{i}
$$

where $q_{i}$ are 'point charges' at position $r_{i}$ and $N$ is the total number of charges used to describe the distribution. This allows the calculation of $R_{\mathrm{g}}$ for any given particle shape, as 
Table 1

Radii of gyration of all initial monodisperse models $\left(R_{\mathrm{g}, \mathrm{id}}\right)$ and of their resulting aDAMs $\left(R_{\mathrm{g}, \mathrm{aDAM}}\right)$ used here.

$R_{\mathrm{g}, \mathrm{id}}$ and $R_{\mathrm{g}, \mathrm{aDAM}}$ were obtained using equations (6) and (8). $\Delta R_{\mathrm{g}}$ is the error between model and fit. In the simulation series of 'cube_da_1-3', the DA size was varied such that the aDAM of 'cube_da_1' has the highest DA diameter of $0.88 \mathrm{~nm}$ (see $\$ 3.1)$.

\begin{tabular}{llll}
\hline Name & Ideal $R_{\mathrm{g}, \text { id }}(\mathrm{nm})$ & $R_{\mathrm{g}, \mathrm{aDAM}}(\mathrm{nm})$ & $\Delta R_{\mathrm{g}}(\%)$ \\
\hline Sphere & 5.8095 & 5.7986 & 0.19 \\
Core-shell & 5.7239 & 5.7697 & 0.80 \\
Ellipsoid & 5.8031 & 5.8059 & 0.05 \\
cube_da_1 & 8.0000 & 8.6659 & 8.32 \\
cube_da_2 & 8.0000 & 7.9348 & 0.80 \\
cube_da_3 & 8.0000 & 7.9739 & 0.33 \\
\hline
\end{tabular}

long as the positions $\mathbf{r}$ of all charges are known. In analogy to equation (7), we define the radius of gyration of an aDAM as

$$
R_{\mathrm{g}, \mathrm{aDAM}}^{2}=\sum_{i}^{N} \operatorname{occ}^{*}\left(\mathbf{x}_{i}\right)\left|\mathbf{x}_{i}\right|^{2} / \sum_{i}^{N} \operatorname{occ}^{*}\left(\mathbf{x}_{i}\right) .
$$

For all monodisperse systems presented in this work, the radius of gyration was calculated analytically for the simulated geometries as well as for the aDAMs using equation (8). As can be seen from the results in Table 1, the deviation of the radii of gyration of the aDAMs from the initial geometry is less than one percent for all simulations with a sufficient large number of DAs (see $\$ 3.1$ for a detailed explanation), justifying this approach. This implies that at least for the presented models there exists some kind of correlation between the initial electron density distribution, from which the theoretical scattering curves were calculated, and the occupancy of the retrieved aDAMs.

\subsection{Linear evaluation}

The size of inorganic NCs is an important tuning knob for various physical properties, especially in the design of novel NC materials. Therefore, one of the fundamental questions regarding the quantitative interpretation of DAMs and aDAMs is the determination of structural dimensions, e.g. to determine the lengths of the sides of a cube along different directions. For DAMs from a single DAMMIN fit, a 'quick' estimate of such dimensions can be given by simply calculating the distance between any two DAs on the model surface.

For the case of aDAMs from multiple fits, the issue of measuring a DAM gets more complex owing to a continuous occupancy transition from particle to solvent. This can be explained by the fact that it is very unlikely that two DAMs obtained from single simulations of the same initial scattering curve will exhibit an identical DA distribution. Therefore, a statistical smearing of the occupancy due to the averaging process will occur near the surface.

For rather isotropic bodies, this issue of a continuous transition region can be avoided by computing occupancy cross sections of the aDAMs, which is achieved by placing a cylinder with a given radius through the centre of mass of the aDAM (see Fig. 1). The DAs within the cylinder are distributed in slices of equal width, such that for each slice a mean occupancy can be calculated by adding up the occupancy values of the DAs inside each slice. Plotting these mean slice occupancy values versus the distance from the aDAM centre results in a linear occupancy profile along a chosen direction of the cylinder axis. Furthermore, the mean slice occupancies between $-R_{\mathrm{g}} / 2<x<R_{\mathrm{g}} / 2$ around the DAM centre are averaged to obtain a mean core occupancy $\overline{\mathrm{occ}}_{\mathrm{c}}$. The transition regions from the particle core to the solvent in the occupancy profile are approximated by a linear fit with the numerical borders of $0.2 \overline{\mathrm{occ}}_{\mathrm{c}}$ and $0.8 \overline{\mathrm{occ}}_{\mathrm{c}}$. From these lines, the halfheight dimension is obtained at $0.5 \overline{\mathrm{occ}}_{\mathrm{c}}$. The result of this procedure for the example of a cube with side length of $16 \mathrm{~nm}$, measured along the 'centre to face-centre' direction, is shown in Fig. 2 (corresponding scattering curves and fits can be found in Fig. S3). As is seen by a comparison of the retrieved aDAM with the ideal initial cube shape (see supplementary Fig. S1), there is clearly a rhombohedral distortion of the cubic shape. This deviation of the corner angles from $90^{\circ}$ was already witnessed by Volkov \& Svergun (2003). However, for a chosen cut-through cylinder radius of $r_{\text {cyl }}=5 d_{\mathrm{DA}}$, where $d_{\mathrm{DA}}$ is the DA diameter of $0.57 \mathrm{~nm}$, a face-to-face distance of $16.2 \mathrm{~nm}$ is measured which is in good agreement with the edge length of the initial cube. It should be noted that with this procedure the

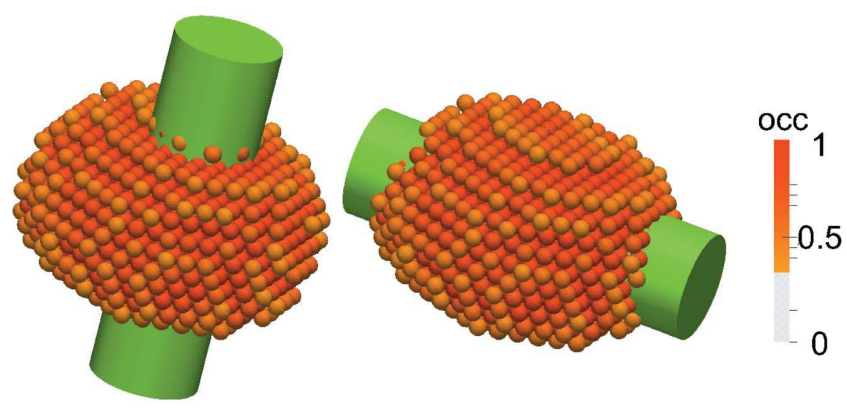

(a)

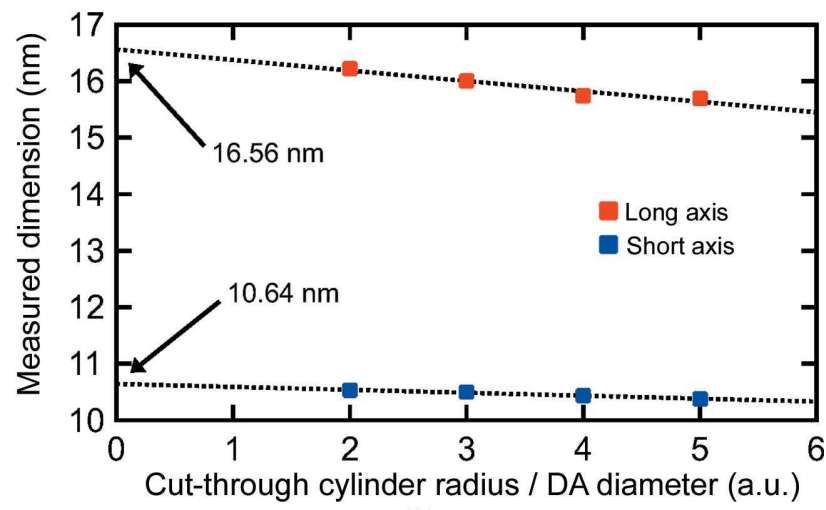

(b)

Figure 1

(a) An aDAM derived from an ellipsoid of revolution with two long axes of radius $8.2 \mathrm{~nm}$ and a short axis of radius $5.25 \mathrm{~nm}$ was used to perform the linear evaluation along the short axis (left) and along the long axis (right). For illustrative reasons all DAs with occ $<1 / 3$ occ $_{\max }$ are not shown. The green cylinder represents the cut-through cylinder with a radius of $3 d_{\mathrm{DA}}$. (b) Linear regression of the obtained measurements as a function of the cut-through cylinder radius. By extrapolating the fitted line to intersect with the ordinate, the real aDAM dimensions of $16.6 / 2=$ $8.3 \mathrm{~nm}$ for the long-axis radius and $10.6 / 2=5.3 \mathrm{~nm}$ for the short-axis radius are derived. 
deviation of the measured distance from the input dimension is significantly less than the resolution defined by the size of the DAs.

The most crucial point of this linear evaluation technique is the transition region from particle core to solvent. For the example presented above, the slices of the cut-through cylinder are always parallel to the face of the cube and, therefore, no distortion of the transition region due to geometrical reasons will occur. If, however, dimensions of curved objects, such as ellipsoids of revolution for example, have to be measured, the result will depend on the size of the cut-through cylinder. This effect could of course be minimized by decreasing the radius. However, this causes a new problem of statistical nature. For small cylinder radii, the probability of finding a DA in every evaluation slice gets low, which results in a strong statistical noise in the obtained profiles. From our investigations we found that, for cylinder radii smaller than $2 d_{\mathrm{DA}}$, it is nearly impossible to evaluate a half-height dimension.

A more promising approach is to measure the dimension of interest multiple times with decreasing cut-through cylinder radii. If the resulting dependence of the measured dimensions as a function of the cylinder radius is approximately linear, an extrapolation to zero cylinder radius can be used to estimate the real size value. This method was applied to the example of an aDAM, obtained from the scattering curve of an ellipsoid of revolution with two long-axis radii of $8.2 \mathrm{~nm}$ and a shortaxis radius of $5.25 \mathrm{~nm}$. The measurements were taken along the long and the short axes of the aDAM [see Fig. 1(a)] where the cylinder radii were chosen to be multiples of $d_{\mathrm{DA}}$. The linear regression is depicted in Fig. 1(b), resulting in short- and long-axis radii of 5.25 and $8.3 \mathrm{~nm}$, respectively, which matches excellently the initial model dimensions.

\subsection{Radial distance distribution}

To obtain information from an aDAM using the linear evaluation method, a decision regarding the measurement

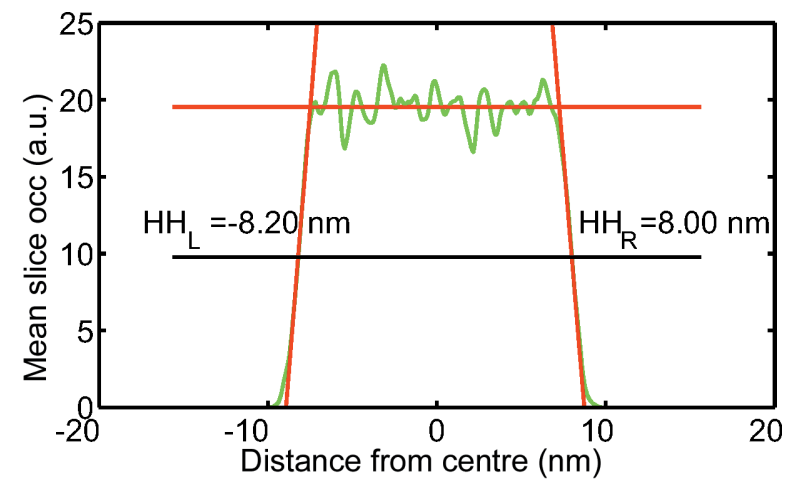

Figure 2

Occupancy profile (green) obtained from the linear evaluation method using a cut-through cylinder perpendicular to the face of the aDAM retrieved from the scattering curve of a cube with side length of $16 \mathrm{~nm}$. The red lines indicate the linear approximations of the occupancy regions. Using the half-height approximation for a cylinder radius of $5 d_{\mathrm{DA}}$, a side length of $16.2 \mathrm{~nm}$ was measured with the corresponding half-height approximation obtained using a cut-through cylinder radius of $5 d_{\mathrm{DA}}$. The result of this procedure yields a side length of $16.2 \mathrm{~nm}$. direction has to be made by the user. To avoid such a preselection, the linear evaluation method was extended by a randomized iteration algorithm, allowing the measurement along all spherical directions. By determining the half-height dimension along a large number of random directions $\left(>10^{3}\right)$, a radial distance distribution $D(r)$ can be obtained, where $r$ is the distance from the centre of the aDAM to the half-height surface. The obtained $D(r)$ can be interpreted as the number of times a given distance was measured, which makes it a generic source of information on the outer particle dimensions. To validate the procedure, this distribution was compared with the $D(r)$ computed from an ideal shape using analytical boundaries. Fig. 3(a) shows the resulting $D(r)$ values for the aDAM of the ellipsoid shown in Fig. 1( $a$ ) for two different cylinder radii as well as the $D(r)$ resulting from an ideal elliptical shape. The two experimental $D(r)$ values are derived from cut-through cylinders with radii of $2 d_{\mathrm{DA}}$ and $5 d_{\mathrm{DA}}$ with $d_{\mathrm{DA}}=0.53 \mathrm{~nm}$. As can be seen, the analytical $D(r)$ and both distance distributions from the aDAM are in

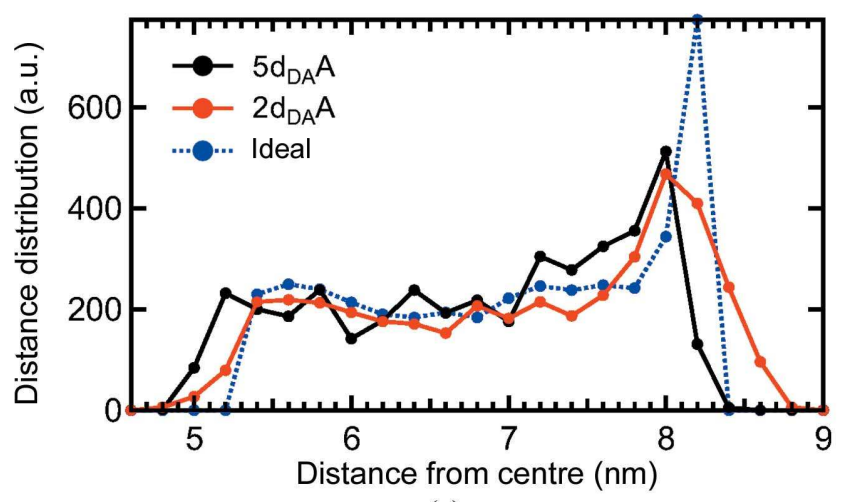

(a)

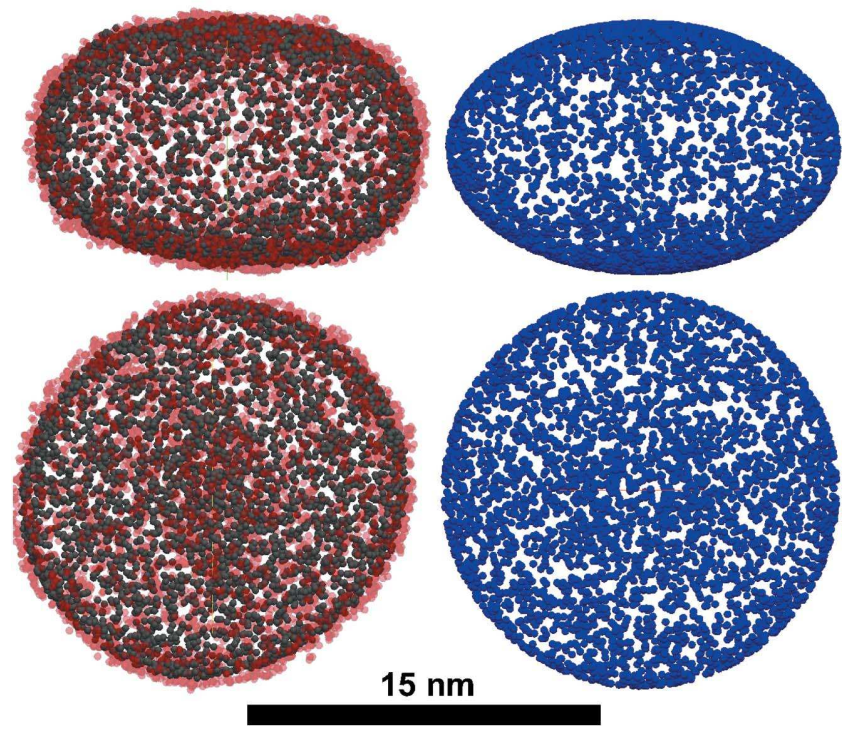

(b)

Figure 3

(a) $D(r)$ of the aDAM shown in Fig. 1 using 4000 randomized directions. The red and the black profiles originate from the same aDAM using different cut-through cylinder sizes. The blue profile was calculated using analytical boundaries with the same dimensions as the initial model with an infinitely thin cylinder. (b) Half-height models from the corresponding distance distributions according to the colour-code shown in $(a)$. 
reasonable agreement. From the two half-height values of the $D(r)$ at the borders, the smallest and largest distances, i.e. the long- and the short-axis dimensions of the ellipsoids, were retrieved. For the $D(r)$ with the smaller cylinder radius of around $1 \mathrm{~nm}$ these values are found to be within $\pm d_{\mathrm{DA}} / 2$ of the initial dimensions, corresponding to a systematic error of only 3.3 and $5.2 \%$ for the long and the short axis, respectively.

As already mentioned above, the difficulty of the interpretation of the aDAMs is the continuous occupancy transition from the aDAM core to the solvent. This is also true for the visualization of the aDAMs because a cutoff occupancy has to be chosen by the user to display a meaningful model, as can be seen in the examples given in Fig. 1. The computation of the $D(r)$ offers an alternative by constructing a half-height surface of the aDAM. This is achieved during the randomization process by plotting the obtained half-height dimensions along each direction. The final outcome is a discrete shape that can now be used to interpret quantitatively the aDAM dimensions. In Fig. 3(b), the half-height surface models for the corresponding $D(r)$ in Fig. 3(a) are shown. For the two halfheight models of the aDAM with different cylinder radii the size effect discussed is also visible, but can be avoided by an extension of the already described linear evaluation method in three dimensions.

\subsection{Spherical average}

Especially for NCs with inhomogeneous electron-density distributions, such as core-shell NCs, not only the outer dimensions but also the inner transition regions from the core to the shell are of interest. To cover such cases, an evaluation procedure based on the 'onion principle' was developed for spherical NCs. Starting from the centre of mass, the average occupancies for spherical shells of a given thickness $t$ are calculated (from our investigations we found $t=R_{\max } / 500$ to be a good compromise between statistical significance and spatial resolution, where $R_{\max }$ is the maximum distance of any DA from the centre of mass found in the aDAM). Therefore, a

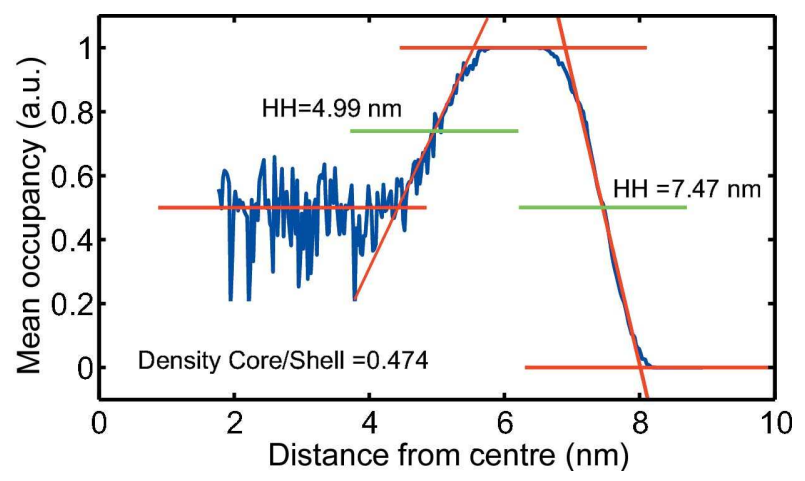

Figure 4

Spherical average profile of an aDAM, which was computed from the initial scattering curve of a spherical core-shell model with a core radius of $5 \mathrm{~nm}$, a shell thickness of $2.5 \mathrm{~nm}$ and an electron density ratio core/ shell $=1 / 2$. The noise close to the aDAM core is caused by the volumetric effect that the number of DAs used to compute the mean occupancy in each shell scales with the third power of the distance from the centre. Numerical errors of the fits were computed and can be found in Table 2. spherically averaged occupancy cross section of the aDAM can be obtained, where the distance between two data points is equal to the shell thickness $t$. Similar to the method discussed above, the transition regions from core to shell as well as from shell to solvent can be represented using linear fits. By manually supplying the fitting borders for the sectionally homogeneous regions, half-height dimensions can be obtained. Furthermore, by comparing the averaged core and shell occupancies, the occupancy ratio between core and shell can be derived. The full procedure was validated for an aDAM resulting from an initial scattering curve of spherical core-shell NCs with a core radius of $5 \mathrm{~nm}$ and a shell thickness of $2.5 \mathrm{~nm}$ with contrast ratio of core $/$ shell $=1 / 2$. The mean occupancy profile as well as the evaluation results are shown in Fig. 4. The obtained results of 4.99 and $7.47 \mathrm{~nm}$ for the core-toshell and shell-to-solvent transition dimensions as well as the core-shell occupancy ratio of 0.474 are in excellent agreement with the initial model dimensions. So far we have only considered monodisperse particles. If inner and outer transition dimensions are to be obtained from aDAMs from real SAS measurements, one has to account for size distributions as well as 'distortions' of the transition region such as facets. For reliable quantitative results, the spherical average evaluation is, therefore, only recommended for monodisperse and spherically symmetric particles. For more complex shapes, such as core-shell cuboids for example, the linear evaluation method can also be used to detect inner transitions, but with lower statistical significance. The mean occupancy values of the outer shells, however, are still of higher statistical accuracy, because a larger number of DAs are found in the outer evaluation shells for geometric reasons. This makes the halfheight results for the nanoparticle surface shells very stable from a numerical point of view and gives very accurate outer particle dimensions.

It has to be noted that all cross sectional evaluation techniques shown above are based on a spherical approach as the reference point is always the centre of mass of the aDAM. This assumption imposes several restrictions and, in particular, excludes these techniques for shapes retrieved from strongly anisotropic and complex particle shapes. Nevertheless, as most inorganic nanocrystals for practical applications exhibit rather isotropic or only slightly anisotropic shapes, the proposed methods may be highly suitable for the evaluation of SAXS data from such systems.

\section{Results and discussion}

\subsection{Model calculations}

The ideal and the retrieved shapes, the scattering curves including the initial scattering intensities, the fitted $p(r)$ and the average of all ten DAMMIN simulation fits performed for homogeneous spheres, cubes, ellipsoids of revolution and core-shell spheres are shown in Figs. S1-S4 as well as in Fig. 5. Quantitative results on these monodisperse models can be found in Tables 1 and 2. Additionally, the normalized spatial discrepancy (NSD) values obtained from the SUPCOMB 
subroutine of DAMAVER of all aDAMs shown in this work are presented in Table S1. Generally speaking, the NSD after the SUPCOMB process is a quantitative estimate of the similarity between three-dimensional objects (Kozin \& Svergun, 2001). In the case of the results shown in Table S1, the average NSD of all possible combinations of DAM pairs that have been used to compute the aDAM is calculated. These values, therefore, represent a measure of the similarity of the individual DAM solutions from the DAM. In a first investigation series, the influence of the DA size was studied in simulations cube_da_1, cube_da_2 and cube_da_3, where the numbers of DAs were set to 2988,5594 and 8853 . For the case of a cube with a side length of $16 \mathrm{~nm}$, this results in DA diameters of $0.88,0.72$ and $0.61 \mathrm{~nm}$. For the case of cube_da_1, we noticed that the scattering curve could not be adequately fitted, as can be seen in Fig. S4. Quantitatively speaking, the $R_{\mathrm{g}}$ of the aDAM as an integral parameter strongly deviates from the initial model, as can be seen in Table 1. In addition to this, the NSD of this aDAM is increased by over $20 \%$ compared with cases with higher numbers of DAs. This can be related to the fact that, with the size of the DAs of around $0.9 \mathrm{~nm}$, the rendering of the sharp edges and corners of the model cube is already insufficient. In all other models, the number of DAs was, therefore, kept to around 6000 DAs.

3.1.1. Shannon channels. With the introduction of DAMMIN for shape retrieval of biological macromolecules, a discussion has arisen regarding the number of SCs that are necessary to obtain reliable results (Koch et al., 2003; Trewhella et al., 2013). The complexity of this discussion can be explained by the fact that several other factors, such as, for example, the accuracy of the experimentally determined data or the available a priori information, can also affect the level of detail that can be gained from a retrieved DAM or aDAM (Koch et al., 2003). Therefore, a systematic study has to be carried out, investigating the direct effects of a decreasing $q$ range on the retrieved DAMs and aDAMs while keeping all other parameters constant. The conclusions drawn from this investigation show possible errors that can occur if DAMs and aDAMS retrieved from experimental SAS data with a restricted number of SCs are over interpreted. Therefore, we started with simulated scattering curves with a varying number of SCs for the core-shell model in Fig. 4, which has a core radius of $5 \mathrm{~nm}$, a shell thickness of $2.5 \mathrm{~nm}$ and a contrast ratio of core/shell $=1 / 2$. These dimensions yield an SC width of $w=$ $\pi / D_{\max }=0.21 \mathrm{~nm}^{-1}$ in the scattering curves as well as in the averaged scattering intensities from ten DAMMIN fits as shown in Fig. 5(a). The NSD values of the retrieved aDAMs can be found in Table S1, but no correlation between the number of SCs and the NSD values could be seen. If, however, the NSD values are computed by comparing an aDAM from polydisperse model data with the aDAM obtained from the monodisperse system, an increase in the NSD can be seen with increasing size distribution (see Table S2). The spherical evaluation method was chosen to further analyse the aDAMs, with the resulting occupancy profiles presented in Fig. 5(b) and the corresponding values in Table 2. A decrease of the $q$ range from 20 to 15 SCs does not significantly impact the
Table 2

Core-shell parameter as a function of the number of Shannon channels $N_{\text {SC. }}$.

Parameters derived from an aDAM of an initial spherical core-shell model with core radius and shell thickness of 5 and $2.5 \mathrm{~nm}$, repectively, and an electron density ratio core/shell $=1 / 2$. Values obtained from the spherical evaluation are the transition dimension from core to shell $\left(x_{\mathrm{cs}}\right)$, the transition dimension from shell to solvent $\left(x_{\mathrm{ss}}\right)$ and the mean occupancy ratio core/shell $\left(r_{\mathrm{c} / \mathrm{s}}\right)$. The provided errors were calculated from standard deviations of the fitting parameters according to error propagation laws.

\begin{tabular}{lllll}
\hline Name & $N_{\mathrm{SC}}$ & $x_{\mathrm{cs}}(\mathrm{nm})$ & $x_{\mathrm{ss}}(\mathrm{nm})$ & $r_{\mathrm{c} / \mathrm{s}}(\%)$ \\
\hline core_shell_1 & 20 & $4.99 \pm 0.44$ & $7.47 \pm 0.13$ & $47.4 \pm 2.3$ \\
core_shell_2 & 15 & $5.01 \pm 0.42$ & $7.47 \pm 0.13$ & $49.2 \pm 2.1$ \\
core_shell_3 & 10 & $4.88 \pm 0.51$ & $7.50 \pm 0.14$ & $51.1 \pm 2.6$ \\
core_shell_4 & 5 & $4.26 \pm 0.76$ & $7.56 \pm 0.21$ & $35.6 \pm 5.9$ \\
\hline
\end{tabular}

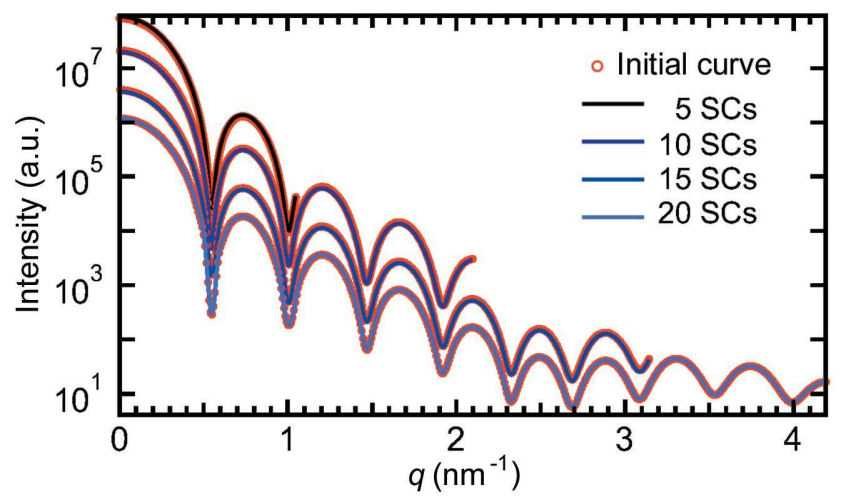

(a)

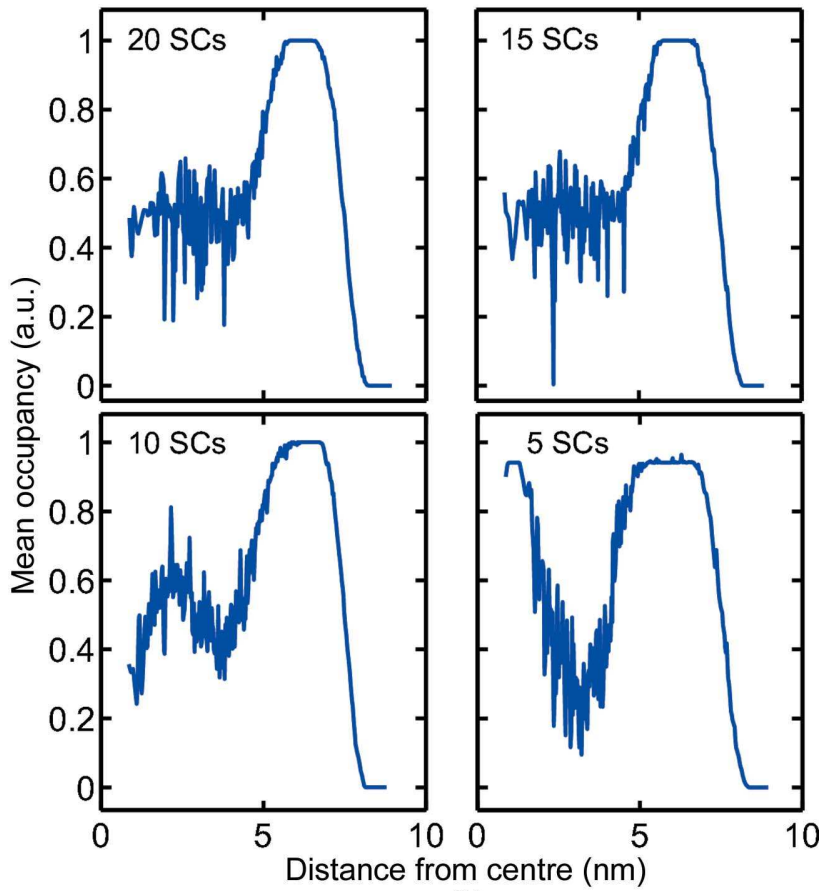

(b)

Figure 5

(a) Scattering curves of the initial core-shell model as used in Fig. 4 with different numbers of SCs. The fitted curves of all ten DAMs used for the computation of the aDAM were averaged and are shown in different colours. (b) Spherical average occupancy cross section of the final aDAMs. For this simple geometry a significant effect can only be witnessed when the number of SCs is decreased to ten or lower. 
results. Only if the $q$ range is further decreased to $10-5$ SCs is a fluctuation of the core occupancy observed, which results in a deviation of the core-shell transition and occupancy ratio values. It should be noted, however, that the outer dimension is not significantly affected even for only five SCs, whereas the core-shell transition region is no longer well defined for SCs $<10$ [see Fig. 5(b)]. This has to be taken into account especially for real SAS data, where it is very difficult to capture the scattering signal with more than 10-15 SCs experimentally. If high-resolution information has to be deduced from the inner density values, the number of SCs should be kept above 10 . For structures, from which only outer dimensions are of interest, lower numbers of SCs may be sufficient.

3.1.2. Polydispersity. In SAXS experiments, smearing effects owing to the experimental setup or the numerical integration of the detector data will occur, which in the case of perfectly monodisperse spheres will mainly result in a smearing of the form factor minima. To account for such

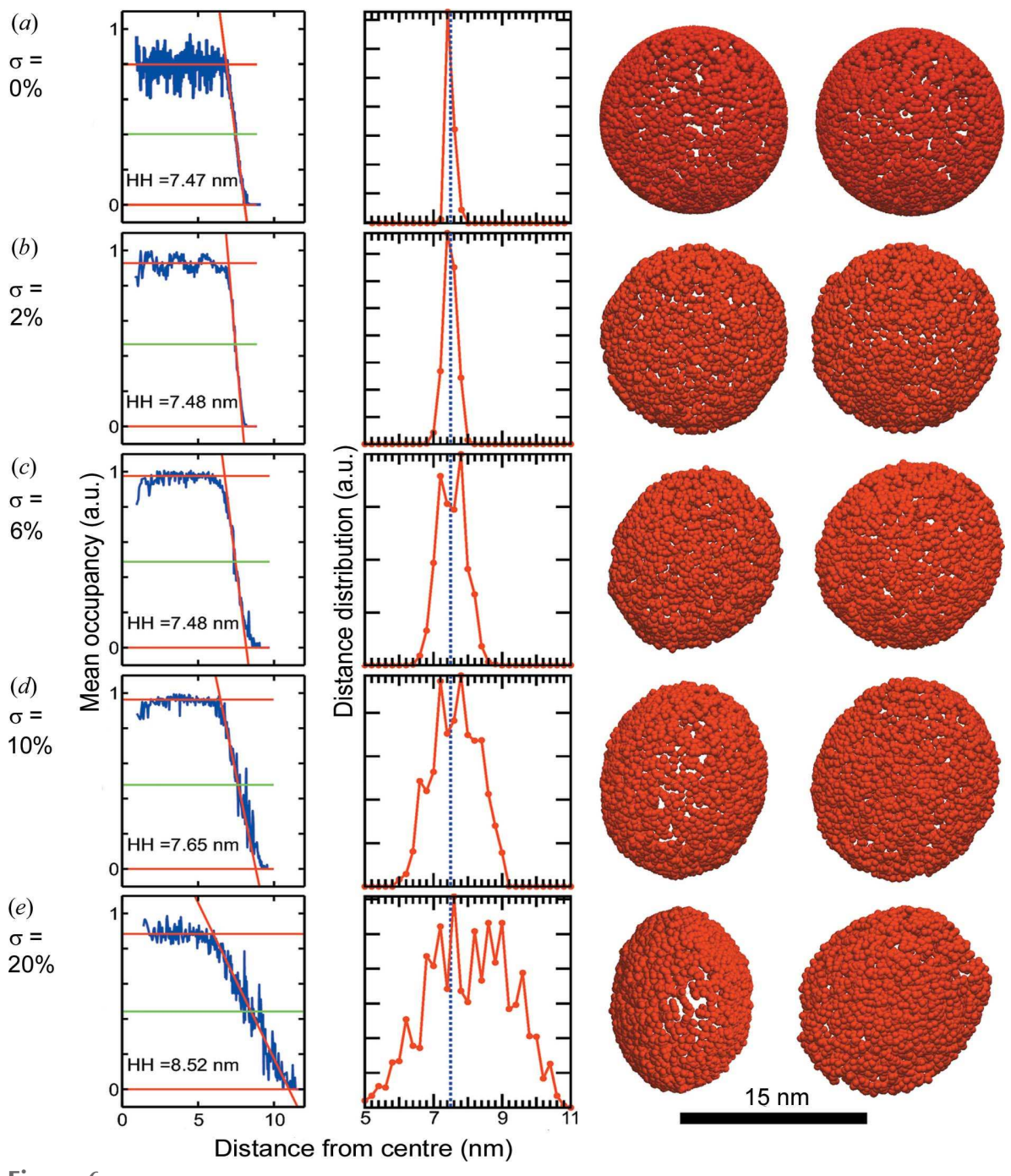

Figure 6

Influence of the size distribution $\sigma$ on spherical aDAMs. The initial scattering curves were calculated according to equation (2) for spheres of mean radius $7.5 \mathrm{~nm}$ (centre of the Gaussian volume distribution). The particle dimensions were derived by using the spherical average evaluation (first column) and the distance distribution $D(r)$ (second column). To visualize the derived shape, the half-height surface is shown from two perpendicular directions (third column). smearing effects, the resolution function of the used experimental setup is needed, which can be employed to desmear the experimental data or to smear the fitting curves with a resolution function (Glatter, 1977; Fratzl et al., 1993; Pedersen et al., 1990). A similar effect can be observed when a small size distribution is introduced into the same system, which therefore makes it sometimes difficult to distinguish between these two effects. In the following, we investigate the influence of smearing on the final aDAMs, where the introduction of a size distribution is used as a measure for the magnitude of the smearing. According to equation (2), scattering curves of polydisperse spheres with a radius of $7.5 \mathrm{~nm}$ were calculated for Gaussian size distributions with standard deviations from 1 to $20 \%$. The evaluation of the aDAMs for $0,2,6,10$ and $20 \%$ is shown in Fig. 6. The NSD values of the retrieved aDAMs, which can be found in Table S1, exhibit no correlation to the magnitude of the size distribution. The quantitative results obtained from the spherical average and $D(r)$ evaluation of the aDAMs can be found in Table 3, while the scattering curves and their corresponding fits are shown in Fig. S2.

Several effects can be observed when the size distribution is increased. (i) The centre of the distance distribution $D(r)$, which corresponds to the mean particle radius, clearly shifts to higher values for a size distribution $\sigma_{\text {init }}>6 \%$. This can be explained by the quadratic correlation between particle volume and scattering intensity according to equation (1). (ii) The slope of the linear fit in the transition region of the spherical average evaluation clearly becomes less steep, which can be interpreted as a smearing of the transition region. This smearing of the transition region can be the result of a local occupancy gradient of the aDAM and also of general deviations of the aDAM from a spherical shape. (iii) The distance distribution $D(r)$ is broadened. By assuming a Gaussian distribution also for $D(r)$, the experimental size distribution $\sigma_{\mathrm{DD}}$ can be back calculated from the full width at half-maximum (FWHM) $w_{\mathrm{DD}}$ of the peak in the $D(r)$ according to $\sigma_{\mathrm{DD}}=2(2 \ln 2)^{1 / 2}\left(w_{\mathrm{DD}} / c_{\mathrm{DD}}\right)$, where $c_{\mathrm{DD}}$ is the peak centre. As can be seen in Table 3, the back-calculated values are in good agreement with the initial input parameters except for the cases $\sigma=0$, $\sigma=1$ and $\sigma=20 \%$. For $\sigma=20 \%$, a complete breakdown of the shaperetrieval method occurs owing to severe smearing. As the back-calculated values show no improvement for polydispersities lower than $\sigma=2 \%$, it can be 
Table 3

Spherical dimensions as a function of the size distribution $\sigma_{\text {init }}$ as obtained from aDAMs of polydisperse homogeneous spheres (radius $7.5 \mathrm{~nm}$ ).

From the spherical average evaluation the half-height values $\mathrm{HH}_{\mathrm{SA}}$ and from the $D(r)$ the peak centre $c_{\mathrm{DD}}$ is obtained corresponding to the sphere radius. FWHM values of the $D(r)$ are used to back calculate the experimental size distribution $\sigma_{\mathrm{DD}}$. The virtual aspect ratio $\mathrm{AR}_{\mathrm{DD}}$ of the $D(r)$ is obtained by using the two half-maximum values.

\begin{tabular}{lllll}
\hline$\sigma_{\text {init }}(\%)$ & $\mathrm{HH}_{\mathrm{SA}}(\mathrm{nm})$ & $c_{\mathrm{DD}}(\mathrm{nm})$ & $\sigma_{\mathrm{DD}}(\%)$ & $\mathrm{AR}_{\mathrm{DD}}$ \\
\hline 0 & $7.47 \pm 0.16$ & 7.49 & 2.2 & 1.04 \\
1 & $7.47 \pm 0.24$ & 7.48 & 2.4 & 1.06 \\
2 & $7.48 \pm 0.19$ & 7.49 & 2.7 & 1.06 \\
4 & $7.49 \pm 0.31$ & 7.52 & 4.1 & 1.10 \\
6 & $7.48 \pm 0.54$ & 7.53 & 5.7 & 1.14 \\
8 & $7.63 \pm 0.42$ & 7.66 & 7.4 & 1.19 \\
10 & $7.65 \pm 0.39$ & 7.75 & 9.2 & 1.24 \\
15 & $7.67 \pm 0.58$ & 8.12 & 12.1 & 1.33 \\
20 & $8.43 \pm 0.45$ & 8.23 & 15.8 & 1.46 \\
\hline
\end{tabular}

concluded that with this value the resolution limit of the aDAMs is reached. This is confirmed by the insufficient fits of the scattering curves for $\sigma_{\mathrm{DD}}<2 \%$ in Fig. S2. Thus, the backcalculated mean deviation $\sigma_{\mathrm{DD}}$ in Table 3 for the case of $\sigma=$ $0 \%$ defines the statistical resolution of the entire shaperetrieval process of the order of $1 \%$. The quantitative results in Table 3 also show that the sphere radius estimated from the spherical average evaluation and from the centre of the $D(r)$ coincide. Considering a DA size of $r_{\mathrm{DA}}=0.25 \mathrm{~nm}$, all outer dimensions obtained from the aDAMs up to $\sigma=10 \%$ are within the interval $7.5 \pm 0.25 \mathrm{~nm}$.

Nevertheless, a closer look at the half-height surfaces of the aDAMs in Fig. 6 rather suggests that with increasing polydispersity the aDAM shape tends to become more and more elliptical. Therefore, a similar series of simulations was performed for the case of polydisperse oblate ellipsoids of revolution to determine a threshold polydispersity above which the initial shape is lost (see Table 4 and Fig. 7 for results). Similar effects to the ones described above for spheres are noticed, such as the clear drift to larger dimensions of the half-height value of the spherical average evaluation. It should be noted that in the case of ellipsoids of revolution the half-height value obtained from the spherical average evaluation does not correspond to a meaningful dimensional value of the model. We have, therefore, set the fitting boundaries to only take the lower part of the occupancy profile into account, such that possible deviations of the occupancy profile from the linear behaviour can be detected (see Fig. 7). Interestingly, for the simulation series dealing with the spherical shape shown in Fig. 6, the spherical average profile never significantly deviates from this tangent fit. For the case of ellipsoids, however, a distinct artefact is seen in the occupancy profile close to the core in the transition region shown in Fig. 7. This 'buckle' in the profile can be observed for all investigated size distributions for ellipsoids, whereas for spheres such a phenomenon cannot be seen at all. This might be a practical way to discriminate spheres from ellipsoids even for small size distributions.

Additionally a broadening of the $D(r)$ is visible, which was also observed for the case of polydisperse spheres. In the case
Table 4

Elliptical dimensions as a function of the size distribution $\sigma_{\text {init }}$ as obtained from aDAMs of ellipsoids of revolution with single- and double-axis radii of 5.25 and $8.5 \mathrm{~nm}$, respectively.

From the spherical average evaluation the half-height values $\mathrm{HH}_{\mathrm{SA}}$ and from the $D(r)$ the peak centre $c_{\mathrm{DD}}$ as well as FWHM $w_{\mathrm{DD}}$ values are obtained. The aspect ratio $\mathrm{AR}_{\mathrm{DD}}$ of the $D(r)$ is obtained by using the two half-maximum values $\mathrm{HH}_{\mathrm{DD}, 1}$ and $\mathrm{HH}_{\mathrm{DD}, \mathrm{r}}$ that correspond to the short and long axis of the ellipsoid of revolution (the aspect ratio of the ideal model is 1.60).

\begin{tabular}{llllll}
\hline$\sigma_{\text {init }}(\%)$ & $\mathrm{HH}_{\mathrm{SA}}(\mathrm{nm})$ & $\mathrm{w}_{\mathrm{DD}}(\mathrm{nm})$ & $\mathrm{AR}_{\mathrm{DD}}$ & $\mathrm{HH}_{\mathrm{DD}, 1}$ & $\mathrm{HH}_{\mathrm{DD}, \mathrm{r}}$ \\
\hline 0 & $7.32 \pm 0.59$ & 2.79 & 1.51 & 5.43 & 8.22 \\
2 & $7.44 \pm 0.67$ & 3.03 & 1.56 & 5.35 & 8.38 \\
6 & $7.57 \pm 0.78$ & 3.19 & 1.59 & 5.35 & 8.54 \\
10 & $7.61 \pm 0.69$ & 2.52 & 1.46 & 5.54 & 8.05 \\
\hline
\end{tabular}

of ellipsoids of revolution, the back calculation of a size distribution from the $D(r)$ is not meaningful because both model shape and size distribution cause a peak broadening. Nevertheless, useful information can be gained from the halfmaximum dimensions or the FWHM of the $D(r)$. In contrast to the conclusions found for spheres, the width seems much less affected by the polydispersity. This can be exemplified by denoting the aspect ratio of the long to the short axis as $A R_{D D}$ in Tables 3 and 4 . Whereas in the case of spheres $A_{D D}$ is strongly affected by the increasing size distribution, in the case of ellipsoids of revolution $\mathrm{AR}_{\mathrm{DD}}$ only increases up to a polydispersity of $6 \%$ and even decreases for the example of $10 \%$. From this it can be concluded that for the example used in this series (initial aspect ratio of double axis/long axis $=1.6$ ) the peak broadening effects seen in the $D(r)$ are clearly
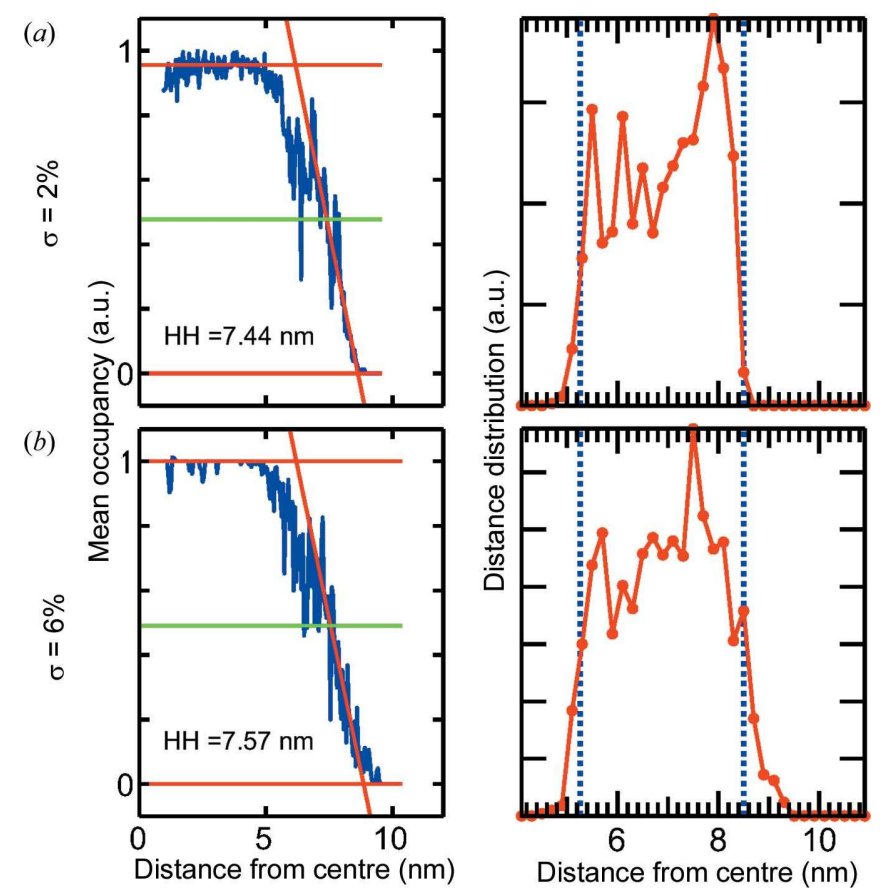

Figure 7

Influence of the size distribution $\sigma$ on elliptical aDAMs. The initial scattering curves were calculated according to equation (2) for ellipsoids of revolution with single- and double-axis radii of 5.25 and $8.5 \mathrm{~nm}$, respectively. The aDAMs were analysed using the spherical average evaluation (first column) and the distance distribution $D(r)$ (second column). 
dominated by the aDAM shape as compared with the influence of the size distribution. But, for the general case of ellipsoids of revolution, the magnitude of the size distribution determines the resolution concerning the model shape.

It was already shown by Mittelbach \& Porod (1962) that it is possible to describe the shape of an arbitrary ellipsoid of revolution by a given synthetic size distribution of spheres. In the case of an ellipsoid of revolution with, for example, axis dimensions $a<b<c$, this size distribution is monomodal with two discontinuities at $a$ and $c$ and a pole at $b$. As a result of these abrupt borders at the discontinuities it is highly unlikely that such a size distribution of spheres will be found in a real system. For polydisperse ellipsoids, however, this distribution will be smeared out to an extent where it eventually could be explained by a real physical system of spheres. If this circumstance is looked at in reciprocal space, this is the rather well known example of the fact that a given scattering curve from a proposed ideal shape can be smeared by either a deviation of this ideal shape or any kind of size distribution. Therefore, for large size distributions the overall shape will essentially be fully lost [which does not exclude that an estimate on particle size can still be made from scattering data (Guinier, 1939)]. However, in the case of small polydispersities the real particle shape will precede such that smearing effects will mainly smudge explicit details of the retrieved shape such as, for example, the surface. As shown above, a similar phenomenon is seen in the spherical average evaluation of the ellipsoids of revolution, where the 'buckle' in the occupancy profile is a strong indication that the $D(r)$ deviates from a typical Gaussian distribution, which would be the case for polydisperse spheres.

In other words: if the size distribution is too broad, or the aspect ratio of the ellipsoid is close to 1 , it is not possible to distinguish if the peak broadening of the $D(r)$ is caused by the size distribution or the shape. The $\mathrm{AR}_{\mathrm{DD}}$ values for the series of polydisperse spheres shown in Table 3 therefore provide a lower threshold aspect ratio of ellipsoids of revolution that could still be resolved.

Our investigations regarding the influence of particle size distributions confirm that, for size distributions of $6 \%$ and smaller, quantitative information on the aDAM dimensions can be gained especially from the distance distributions. The obtained particle dimensions in this range were within an accuracy of $\pm d_{\mathrm{DA}}$. To estimate a statistical error the obtained dimensions were compared with the initial values. For size distributions below $8 \%$ even the largest deviation found was only $3 \%$. Larger deviations for both sphere and ellipsoids of revolution were only witnessed for $\sigma \geq 10 \%$. But, even in the case of an ellipsoid with $\sigma=10 \%$, the statistical error was only at $5 \%$. In the case of spheres with a $\sigma \geq 15 \%$, the error of the dimensional values obtained was found to be between 8 and $10 \%$.

\subsection{Real nanoparticle system}

To provide an experimental validation of the interpretation methods presented in this work, a full shape-retrieval proce- dure was conducted for bismuth NCs. The NCs were synthesized according to the method given by He et al. (2015). TEM images [see inset of Fig. 8(a)] of evaporation dried NCs suggest a spherical shape with a diameter of $14 \mathrm{~nm}$. SAXS experiments of $\mathrm{Bi} \mathrm{NCs}$ diluted in toluene were performed on a NanoStar laboratory system (Bruker AXS, Karlsruhe) using $\mathrm{Cu} K \alpha$ radiation. The experimentally accessible $q$ range is equivalent to approximately $12 \mathrm{SCs}$. The resolution function of the setup was measured using rat-tail tendons (Fratzl et al., 1992). After background subtraction, the indirect Fourier transform was calculated using GNOM. From the $p(r)$ function, a radius of gyration of $5.32 \mathrm{~nm}$ was obtained. The desmeared scattering data were further used for computation of the DAMs and subsequently of the aDAM using an initial spherical volume with a diameter of $17 \mathrm{~nm}$. As suggested above, the DA size was chosen to result in approximately 6000 DAs, which leads to a DA diameter of $0.43 \mathrm{~nm}$. The experimental scattering curve, the $p(r)$ in real space with its corresponding scattering intensity in reciprocal space and the averaged scattering intensity of all ten DAMMIN fits can be found in Fig. 8(a). The weighted radius of gyration of the aDAM was computed according to equation (8), which resulted in $5.29 \mathrm{~nm}$ [deviation of $0.56 \%$ from the value obtained from the $p(r)]$. The spherical average evaluation as shown in Fig. $8(b)$ yields a half-height dimension of $6.56 \mathrm{~nm}$. The distance distribution $D(r)$ was obtained using 4000 random directions and can be found in Fig. 8(c). From this, half-maximum dimensions of 6.09 and $7.06 \mathrm{~nm}$ were measured, which results in an aspect ratio of 1.16. From to the data in Table 3, this peak broadening could be caused by either spheres with a size distribution of approximately $6.3 \%$ [from the FWHM of the $D(r)$ ] or an ellipsoid of revolution with the given aspect ratio. In the spherical averaged profile, a similar 'buckle' close to the linear core-surface transition region can be seen. A similiar behaviour was observed before in the case of ellipsoids of revolution (Fig. 7) and not in the case of spheres (Fig. 6). Interestingly, a closer look to the mean shape represented by the half-height surface in Fig. 8(d) reveals faint facets. To test the hypothesis of a polyhedral faceted shape, model form factors were computed for both polydisperse spheres and polydisperse ellipsoids, using the GIFT software (Bergmann et al., 2000). Subsequently, the form factors of both models were then used to fit corresponding Gaussian size distributions weighted by particle volume. This resulted in a polydispersity of $8.9 \%$ for spheres and $2.3 \%$ for ellipsoids of revolution, each with the dimensions obtained from the $D(r)$. However, as can be seen in Fig. 8(e) the measured scattering curve can not be described satisfactorily by these models, which implies that the real mean NC shape deviates from these simplified model geometries.

The remaining difference can, therefore, be most probably attributed to an elliptical and faceted particle shape as suggested from the retrieved half-height surface models shown in Fig. $8(d)$. From the three-dimensional mean shape model as well as from the $D(r)$ analysis, we derive a short axis and long axis of 6.1 and $7.1 \mathrm{~nm}$, respectively. The planar faceted shapes of some single NCs are also visible in the TEM 


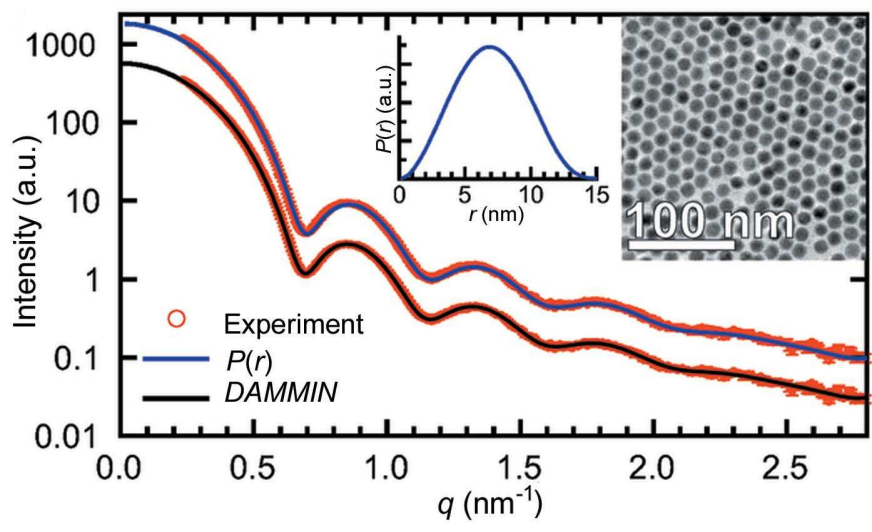

(a)

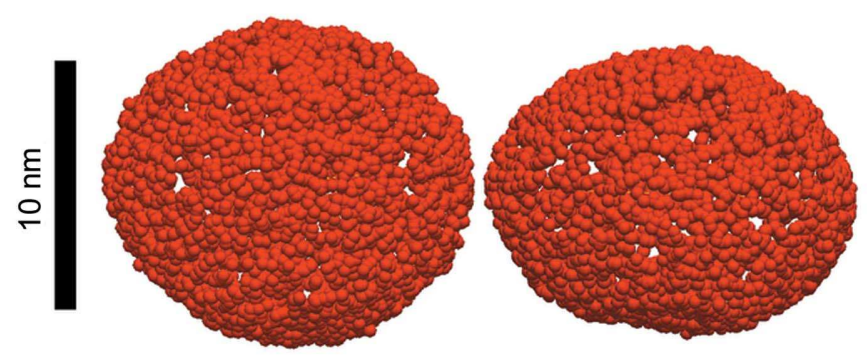

(d)

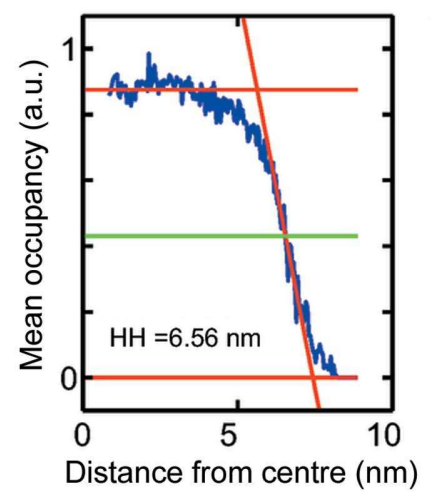

(b)

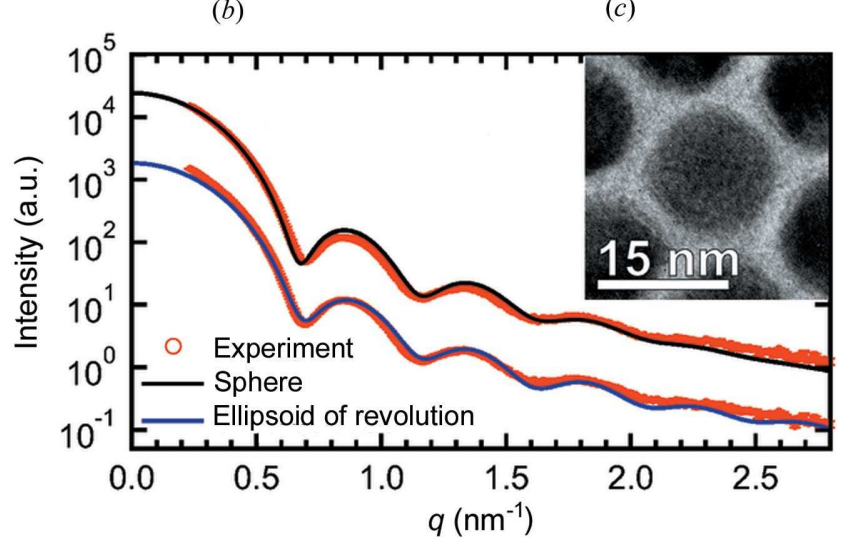

(e)

Figure 8

(a) Experimental scattering curve (red) of Bi NCs with a suggested diameter from the TEM image in the inset of around $14 \mathrm{~nm}$. The $p(r)$ was obtained using GNOM (blue) and further used for the DAMMIN simulations, where the scattering curves of ten fits were averaged (black). The aDAM was evaluated using the spherical average evaluation $(b)$. From the $D(r)$ shown in $(c)$, half-maximum values of 6.09 and $7.06 \mathrm{~nm}$ were obtained, corresponding to spheres with a polydispersity of approximately $6.4 \%$ or ellipsoids of revolution with an aspect ratio of 1.16 . The derived shape is represented using the half-height surface in $(d)$. (e) Using the obtained values from the $D(r)$, analytical form factors are calculated to fit the experimental scattering curve assuming a Gaussian size distribution. This resulted in a polydispersity of $8.9 \%$ in the case of spheres (black) and 3.2 in the case of the elliptical model (blue). Compared to the experimental data (red) the elliptical model is in good agreement. Remaining deviations can be attributed to a faceted shape, as seen for single NCs [(e) inset].

image shown in the inset of Fig. 8(e). Obviously, this faceted three-dimensional mean shape, as shown in Fig. $8(d)$, is necessary to retrieve the very good agreement between the experimental scattering curve and the DAMMIN fits shown in Fig. 8(a). In other words, DAM and aDAM shape retrieval using $D A M M I N$ and $D A M A V E R$ is also able to detect facets in the mean shape of slightly polydisperse NCs, whereas standard fitting procedures can not resolve such details.

\section{Conclusions}

The $a b$ initio shape-retrieval method DAMMIN was applied to calculated scattering curves from model systems similar to those found for inorganic nanocrystals. The coalescence of several single DAMs to one averaged dummy atom model results in a three-dimensional DA density distribution, which exhibits a sensitivity to the polydispersity of the real particle system. It is shown for several geometries that the radius of gyration of the mean DA occupancy obtained from the aDAMs agrees well with the radius of gyration calculated directly from the original model particles. We further implemented and validated techniques based on known cross sectional evaluation methods that can be used to measure distinct dimensions of the aDAMs. A special focus was set on the evaluation of isotropic shapes such as those commonly found in real nanocrystal systems. The normalized spatial discrepancy values alone from the aDAMs did not allow the influence of the accessible scattering angle range to be studied quantitatively. The presented evaluation methods, however, clearly showed a loss of information for the investigated NC shapes with a decreasing number of Shannon channels. Especially if core-shell particles are to be studied, at least 10$15 \mathrm{SCs}$ are recommended to also resolve details about the core. Furthermore, the effects of an increasing size distribution on the final aDAMs were investigated. Smearing equivalent to size distributions up to $6 \%$ shows very little effect on the final aDAM, i.e. the error of the retrieved dimensions is below $3 \%$. Above $10 \%$, the retrieved shapes start to deviate from the initial models, but nevertheless the quantitatively obtained particle dimensions deviate only 8$10 \%$ from the initial values, even for size distributions up to $20 \%$. By applying the knowledge obtained from these theoretical polydisperse models, a full reconstruction procedure was successfully performed to retrieve the shape and the size 
distribution of bismuth NCs without the use of any previous assumptions on the particle shape.

\section{Acknowledgements}

The authors thank Thomas Antretter from the Institute of Mechanics, Montanuniversitaet Leoben for the computational resources. The evaluation methods were written for MATLAB and are available upon request from the corresponding author. MK acknowledges financial support by the Swiss National Science Foundation (SNF, project 200021_140245).

\section{References}

Ahrenkiel, S., Yu, P., Murphy, J., Nedeljković, J. \& Donohoe, B. (2008). J. Microsc. 230, 382-387.

Alves, C., Pedersen, J. S. \& Oliveira, C. L. P. (2014). J. Appl. Cryst. 47, 84-94.

Bergmann, A., Fritz, G. \& Glatter, O. (2000). J. Appl. Cryst. 33, 1212 1216.

Blanchet, C. \& Svergun, D. (2013). Annu. Rev. Phys. Chem. 64, 37-54.

Caruge, J. M., Halpert, J. E., Wood, V., Bulović, V. \& Bawendi, M. G. (2008). Nat. Photon. 2, 247-250.

Chacón, P., Morán, F., Díaz, J., Pantos, E. \& Andreu, J. (1998). Biophys. J. 74, 2760-2775.

Debye, P. (1915). Ann. Phys. 351, 809-823.

Fratzl, P., Groschner, M., Vogl, G., Plenk, H., Eschberger, J., FratzlZelman, N., Koller, K. \& Klaushofer, K. (1992). J. Bone Miner. Res. 7, 329-334.

Fratzl, P., Langmayr, F. \& Paris, O. (1993). J. Appl. Cryst. 26, 820-826. Glatter, O. (1977). J. Appl. Cryst. 10, 415-421.

Guinier, A. (1939). Ann. Phys. (Paris), 12, 161-237.

Gur, I., Fromer, N., Geier, M. \& Alivisatos, A. (2005). Science, 310, 462-465.

He, M., Protesescu, L., Caputo, R., Krumeich, F. \& Kovalenko, M. V. (2015). Chem. Mater. 27, 635-647.

Hens, Z., Vanmaekelbergh, D., Stoffels, E. \& van Kempen, H. (2002). Phys. Rev. Lett. 88, 236803.

Huang, M. H. \& Lin, P. H. (2012). Adv. Funct. Mater. 22, 14-24.

Kirkpatrick, S., Gelatt, C. D. J. \& Vecchi, M. P. (1983). Science, 220, 671-680.

Koch, M., Vachette, P. \& Svergun, D. (2003). Q. Rev. Biophys. 36, 147227.
Kohlbrecher, J. (2008). SASfit. Laboratory for Neutron Scattering, Paul Scherrer Institut, Villigen, Switzerland.

Kozin, M. B. \& Svergun, D. I. (2001). J. Appl. Cryst. 34, 33-41.

Lechner, R. T., Fritz-Popovski, G., Yarema, M., Heiss, W., Hoell, A., Schülli, T. U., Primetzhofer, D., Eibelhuber, M. \& Paris, O. (2014). Chem. Mater. 26, 5914-5922.

Márquez, J. A., Smith, C., Petoukhov, M., Lo Surdo, P., Mattsson, P., Knekt, M., Westlund, A., Scheffzek, K., Saraste, M. \& Svergun, D. (2003). EMBO J. 22, 4616-4624.

Midgley, P. \& Weyland, M. (2003). Ultramicroscopy, 96, 413-431.

Mittelbach, P. \& Porod, G. (1962). Acta Phys. Austriaca, pp. 122-147.

Moore, P. B. (1980). J. Appl. Cryst. 13, 168-175.

Nair, G., Chang, L., Geyer, S. \& Bawendi, M. (2011). Nano Lett. 11, 2145-2151.

Pedersen, J. S. (1997). Adv. Colloid Interface Sci. 70, 171-210.

Pedersen, J. S., Posselt, D. \& Mortensen, K. (1990). J. Appl. Cryst. 23, 321-333.

Reiss, P., Protiere, M. \& Li, L. (2009). Small, 5, 154-168.

Robel, I., Subramanian, V., Kuno, M. \& Kamat, P. V. (2006). J. Am. Chem. Soc. 128, 2385-2393.

Rogach, A., Gaponik, N., Lupton, J., Bertoni, C., Gallardo, D. E., Dunn, S., Li Pira, N., Paderi, M., Repetto, P., Romanov, S. G., O'Dwyer, C., Sotomayor Torres, C. M. \& Eychmüller, A. (2008). Angew. Chem. Int. Ed. 47, 6538-6549.

Rubinstein, M. \& Colby, R. (2003). Polymer Physics. Oxford University Press.

Shannon, C. E. (1948). Bell System Tech. J. 27, 379-423.

Shtykova, E., Huang, X., Remmes, N., Baxter, D., Stein, B., Dragnea, B., Svergun, D. \& Bronstein, L. (2007). J. Phys. Chem. C, 111, 18078-18086.

Shtykova, E., Kuchkina, N., Shifrina, Z., Bronstein, L. \& Svergun, D. (2012). J. Phys. Chem. C, 116, 8069-8078.

Svergun, D. I. (1992). J. Appl. Cryst. 25, 495-503.

Svergun, D. I. (1999). Biophys. J. 76, 2879-2886.

Svergun, D., Petoukhov, M. \& Koch, M. (2001). Biophys. J. 80, 29462953.

Trewhella, J., Hendrickson, W. A., Kleywegt, G. J., Sali, A., Sato, M., Schwede, T., Svergun, D. I., Tainer, J. A., Westbrook, J. \& Berman, H. M. (2013). Structure, 21, 875-881.

Volkov, V. V. \& Svergun, D. I. (2003). J. Appl. Cryst. 36, 860-864.

Walther, D., Cohen, F. E. \& Doniach, S. (2000). J. Appl. Cryst. 33, 350-363.

Wehrenberg, B., Wang, C. \& Guyot-Sionnest, P. (2002). J. Phys. Chem. B, 106, 10634-10640. 